\title{
Sex Effect on Productive Parameters, Carcass and Body Fat Composition of Two Commercial Broilers Lines
}

Author(s)

Rondelli $S^{1}$

Martinez $\mathrm{O}^{1}$

García PT ${ }^{2}$

1 Universidad Nacional de Luján (Bs. As. - Argentina)

2 ITA CA CNIA - INTA CASTELAR

(Bs. As. - Argentina)

Mail Address

C.Correo N 284

Luján

Buenos Aires, Argentina

E-mail: omartin@s6.coopenet.com.ar

\section{Keywords}

Broilers, cholesterol, fatty acids, lines.

\section{ABSTRACT}

The aim of this work was to evaluate sex effect on behavior of two commercial broilers lines. Productive parameters, carcass composition and yield, amount and quality of fat deposited in the meat and skin and total body fat were studied. A completely randomized design with four treatments (MR-Male Ross, HR-Female Ross, MAF-Male Avian Farm and HAF-Female Avian Farm) and eight repetitions of 40 chickens was used. Animals received water and food ad libitum. After 50 days, two birds (a male and a female) per repetition were chosen at random and slaughtered for carcass evaluation. Samples of breast, leg, skin and abdominal fats were analyzed to determine the percentage of intramuscular fat, total cholesterol and fatty acid composition. The Ross line showed higher final weight and weight gain, better intake and feed conversion rate. Also, it was observed that females of both lines had similar results in relation to these parameters. Better yield of breast with bone was found in Ross males and females. In relation to legs and thigh, Ross males showed better results and no differences were observed among females of both lines. Males deposited less fat than females. Finally, differences in percentage of intramuscular fat, content of cholesterol in breast with skin, leg with skin and skin, percentage of saturated, mono and polyunsaturated fatty acids were observed between sex and lines.

\section{INTRODUCTION}

The great scientific and technological development of poultry industry in the last years demanded the evaluation of different commercial broiler lines, as well as different handling techniques, in order to improve production efficiency and help in decision-making. In a world scale, there is a great tendency for increasing chicken meat consumption (Mendes et al., 1988).

In relation to nutrition, it is essential to know clearly and precisely the requirements of the animal species according to their production potential, physiological characteristics, anti-nutritional factors, among others. Adequate choice of foods and diet formulation help not only to reach nutritional requirements but also to increase the levels of feed conversion (Masato Morita, 1992).

Carcass composition can be modified by age, sex, handling and diet manipulation, and it is known that fat deposition increases with age. This is simply related to maturity and happens in the majority of species (Leeson, 1995).

The main reason of absence of uniformity in sex-mixed flocks is the sexual dimorphism of meat type chicken. In face of the necessity of handling birds in homogeneous groups, poultry farmers have adopted single-sex lots in finishing broilers. This difference in growth rate is evidenced in carcass conformation. Most studies report that females show 
higher breast proportion than males, but lower leg and thigh proportion (Lazzari \& Paganini, 1999). Mallo et al. (1997) evaluated the commercial lines Anak and Ross using a diet with low density of nutrients in all treatments. It was reported higher body, carcass and breast weights in broilers from Ross line whereas higher abdominal fat deposition was observed in Anak line chickens. Souza et al. (1995) evaluated carcass traits of four commercial broiler lines, Arbor Acres, Hubbard, Cobb and Ross under the same nutritional management and found higher leg and thigh yield in Hubbard males and higher breast yield in Ross line. Female birds will always be fatter than males because female hormones stimulate fat deposition. From a practical point of view, these differences limit the age in which females can be commercialized (Leeson, 1995). Fat amount, fat quality and cholesterol content in food are important to be considered when the relationship between fat and the risk of some cardiovascular diseases and cancer is evaluated, and animal nutrition, sex and lines can affect carcass fat composition (Cherian et al., 1996; Ahn et al., 1995).

Based on these sex and lines characteristics this study was performed aiming to determine the effect of sex on productive parameters, carcass composition and amount and quality of body fat in two commercial broiler lines.

\section{MATERIAL AND METHODS}

de Luj

Experiment was carried out at Universidad Nacional the amount and quality of carcass fat were performed in ITA CA CNIA INTA Castelar.

Mobile experimental cages with $4 \mathrm{~m}^{2}$ were used. Animal groups and feed were weighed weekly to determine live weight, weight gain, daily intake and weekindex per bird per day. The experimental diets used in this study (Table 1) and water were offered ad libitum.

At the end of the experimental period (50 days), a male and a female of each repetition (16 birds per treatment) were randomly chosen, and after 12 hours of fasting the birds were weighed and slaughtered for carcass evaluation. Carcasses were chilled for further analysis.

Live weight, carcass weight and weights of breast without bone and skin, leg, thigh and abdominal fat were obtained. The data were submitted to analysis variance in a $2 \times 2$ completely randomized factorial design, with 4 treatments (MR - male Ross; $\mathrm{MH}$ - female Ross; MAF -males Avian and HAF - females Avian Farms) and 8 repetitions of 40 birds each.

\begin{tabular}{|c|c|c|c|}
\hline Ingredients (\%) & $\begin{array}{c}\text { Starter } \\
0-21 \text { days }\end{array}$ & $\begin{array}{c}\text { Grower } \\
22-35 \text { days }\end{array}$ & $\begin{array}{c}\text { Finisher } \\
36 \text { - } 49 \text { days }\end{array}$ \\
\hline Fish meal & 2.00 & 0.20 & 0.20 \\
\hline Meat meal & 5.70 & 8.89 & 5.00 \\
\hline Sand & 0.35 & 0.35 & 0.00 \\
\hline Oyster shell & 0.00 & 0.01 & 0.00 \\
\hline DL-Methionine & 0.12 & 0.13 & 0.09 \\
\hline Bicalcium phosphate & 3.52 & 3.10 & 2.22 \\
\hline Fat & 0.01 & 0.10 & 0.00 \\
\hline Bone Ash & 0.01 & 0.25 & 1.33 \\
\hline L-Lysine & 0.05 & 0.16 & 0.03 \\
\hline Ground corn & 53.31 & 58.44 & 62.41 \\
\hline $\operatorname{Mix} V+M^{1}$ & 0.35 & 0.35 & 0.35 \\
\hline Sodium chloride & 0.35 & 0.30 & 0.30 \\
\hline Soybean meal & 23.43 & 20.48 & 25.62 \\
\hline Gluten & 0.00 & 1.24 & 0.00 \\
\hline Wheat & 6.00 & 6.00 & 2.64 \\
\hline Inert & 4.81 & 0.00 & 0.00 \\
\hline Total & 100 & 100 & 100 \\
\hline \multicolumn{4}{|c|}{ Calculated values (\%) } \\
\hline Fat & 3.7 & 4.6 & 6.0 \\
\hline Ash & 5.0 & 4.7 & 4.5 \\
\hline Calcium & 0.95 & 0.85 & 0.85 \\
\hline Phosphorus & 0.76 & 0.67 & 0.68 \\
\hline Metabolizable Energy (Kcal/kg & g) 2915 & 3100 & 3230 \\
\hline Lysine & 1.03 & 1.06 & 0.95 \\
\hline Methionine & 0.45 & 0.47 & 0.41 \\
\hline Met+Cys & 0.76 & 0.79 & 0.72 \\
\hline \multicolumn{4}{|c|}{ 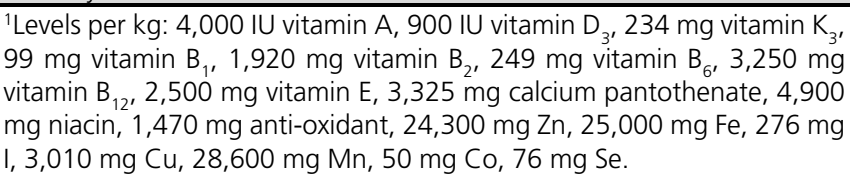 } \\
\hline
\end{tabular}

Samples of breast, leg, skin and abdominal fat were analyzed for percentage of fat, fatty acid composition and cholesterol content (COL). Lipids were extracted as describe by Folch et al. (1957).

Fatty acid composition was determined by methylesters analysis using a GLC with WCOT column with $50 \mathrm{~m}$ melted silicium CP-SIL88 (García et al., 1999), and cholesterol content was determined by the method described by Roschlan et al. (1975). Data were analyzed by General Linear Models (SAS, 1998).

\section{RESULTS AND DISCUSSION}

Weekly body weight and weight gain for both lines are shown in Table 2 . The statistical analysis of the data revealed that after 28 days of life (4 weeks), significant differences $(p<0.05)$ in live weight and weight gain were seen among treatments, with lower live weights being observed for the birds of MR and HR treatments $(p<0.05)$. Weight gain during the last three weeks of experiment was different $(p<0.05)$ between males, 


\begin{tabular}{|c|c|c|c|c|c|}
\hline & \multirow[b]{2}{*}{ Age (week) } & \multicolumn{2}{|c|}{ Body weight (g) } & \multicolumn{2}{|c|}{ Weight gain $(\mathrm{g})$} \\
\hline & & ROSS & Avian Farms & $\overline{\text { ROSS }}$ & Avian Farms \\
\hline & 1 & $136.7 a^{1}$ & $136.2 \mathrm{a}$ & $92.4 \mathrm{a}$ & $85.7 \mathrm{a}$ \\
\hline & 2 & $342.2 \mathrm{a}$ & $330.6 a b$ & $205.4 a$ & $187.2 \mathrm{ab}$ \\
\hline & 3 & $730.0 \mathrm{a}$ & $691.1 \mathrm{~b}$ & $387.8 a$ & $368.3 a b$ \\
\hline \multirow{7}{*}{ Males } & 4 & $1,314.2 \mathrm{a}$ & $1,263.6 c$ & $584.3 \mathrm{a}$ & 495.9 b \\
\hline & 5 & $1,939.2 \mathrm{a}$ & $1,847.1 \mathrm{C}$ & $625.0 \mathrm{a}$ & $549.7 b$ \\
\hline & 6 & $2,518.7 \mathrm{a}$ & $2,394.4 c$ & $579.5 a$ & 508.8 b \\
\hline & 7 & $3,209.1 \mathrm{a}$ & $3,068.4 c$ & $690.4 \mathrm{a}$ & $509.1 \mathrm{~b}$ \\
\hline & 1 & $139.1 \mathrm{a}$ & $132.2 \mathrm{a}$ & $80.2 \mathrm{a}$ & $82.3 \mathrm{a}$ \\
\hline & 2 & $326.3 a b$ & $305.8 \mathrm{~b}$ & $194.4 \mathrm{ab}$ & $173.5 b$ \\
\hline & 3 & $694.6 \mathrm{ab}$ & $648.8 \mathrm{C}$ & 360.4 b & $343.0 \mathrm{~b}$ \\
\hline \multirow[t]{4}{*}{ Females } & 4 & $1,190.4 b$ & $1,082.7 d$ & $572.7 \mathrm{a}$ & $434.0 \mathrm{c}$ \\
\hline & 5 & $1,740.1 \mathrm{~b}$ & $1,623.3 d$ & $583.5 \mathrm{ac}$ & 540.6 bc \\
\hline & 6 & $2,248.95 b$ & $2,099.3 d$ & 508.8 bc & $476.0 \mathrm{~b}$ \\
\hline & 7 & $2,758.03 \mathrm{~b}$ & $2,635.6 \mathrm{~d}$ & $672.0 \mathrm{a}$ & $536.4 b$ \\
\hline
\end{tabular}

1 - For each independent parameter, similar letters within a same row are not statistically different $(p>0.05)$.

but not between females; however, at 42 days of age, difference in body weight was found $(p<0.05)$ between Ross and Avian Farm females.

Feed intake during the whole experimental period is shown in Table 3. The data revealed that, as expected, male birds ate more than female. Between lines, the findings showed a difference $(p<0.05)$ after 28 days of life, with male and female of Ross line eating more than Avian Farms.

Feed conversion index of Ross males and females was lower $(p<0.05)$ than conversion of birds from the Avian Farm (Table 3). A significant difference in carcass weight was noticed between sex and lines (Table 4). Birds from MR treatment had higher carcass weight; and females showed higher percentage of breast than males, which was already expected (Lázzari \& Paganini, 1999). There was no significant difference in the thigh and leg percentage between females. On the other hand, males showed higher thigh and leg proportion than females, similarly to results reported by Lázzari \& Paganini (1999) and $\mathrm{M}$ endes et al. (1988). There were no significant differences in abdominal fat, but higher deposition of cloacal fat was seen in females of both lines than in males. This tendency persisted and values of total fat were similar to those found by Leeson (1995).

Table 5 shows the percentage of fat in breast and legs without skin, as well as skin fat percentage. Birds from HR and MR treatments had lower fat content in breast and higher in leg than HAF and MAF. Females from both lines had fatter skin than males. Cholesterol

\begin{tabular}{|c|c|c|c|c|c|}
\hline & \multirow[b]{2}{*}{ Age (week) } & \multicolumn{2}{|c|}{ Feed intake $(\mathrm{g})^{1}$} & \multicolumn{2}{|c|}{ Feed conversion index } \\
\hline & & ROSS & Avian Farms & ROSS & Avian Farms \\
\hline & 1 & $99.6 a^{2}$ & $92.6 \mathrm{ab}$ & 0.730 & 0.662 \\
\hline & 2 & $374.6 a b$ & $387.6 \mathrm{a}$ & 1.099 & 1.190 \\
\hline & 3 & 968.1 a & $942.0 \mathrm{ab}$ & 1.327 & 1.358 \\
\hline \multirow[t]{7}{*}{ Males } & 4 & $1,882.6 \mathrm{a}$ & $1,764.7 \mathrm{~b}$ & $1.433 \mathrm{a}$ & $1.483 a b$ \\
\hline & 5 & $3,109.8$ a & $2,877.1 \mathrm{~b}$ & 1.604 & 1.654 \\
\hline & 6 & $4,448.3 a$ & $4,062.3 b$ & 1.766 & 1.807 \\
\hline & 7 & $6,000.8 a$ & $5,480.1 \mathrm{~b}$ & $1.870 \mathrm{~b}$ & $1.988 a$ \\
\hline & 1 & $88.4 \mathrm{ab}$ & $82.2 \mathrm{~b}$ & 0.649 & 0.620 \\
\hline & 2 & 375.3 a & $357.7 \mathrm{~b}$ & 1.138 & 1.170 \\
\hline & 3 & $896.6 b c$ & $864.1 \mathrm{c}$ & 1.299 & 1.330 \\
\hline \multirow[t]{4}{*}{ Females } & 4 & $1,769.0 \mathrm{~b}$ & $1,643.3 \mathrm{c}$ & $1.414 \mathrm{a}$ & $1.520 \mathrm{~b}$ \\
\hline & 5 & $2,877.1 \mathrm{~b}$ & $2,986.7 \mathrm{~d}$ & 1.604 & 1.660 \\
\hline & 6 & $4,267.8 \mathrm{C}$ & $3,807.7 d$ & 1.781 & 1.810 \\
\hline & 7 & $5,8075 \mathrm{c}$ & $5,224.1 d$ & $1.893 a$ & $1.980 \mathrm{~b}$ \\
\hline
\end{tabular}

\footnotetext{
1 - Accumulated feed intake. 2 - For each independent parameter, similar letters within a same row are not statistically different ( $p>0.05)$.
} 


\begin{tabular}{|c|c|c|c|c|c|c|c|c|c|c|c|c|c|}
\hline & \multirow{3}{*}{$\begin{array}{l}\text { Body Weight } \\
\text { (g) }\end{array}$} & \multicolumn{2}{|c|}{$C$ arcass } & \multicolumn{4}{|c|}{ Breast with bone Thigh and leg } & \multicolumn{6}{|c|}{ Fat content } \\
\hline & & \multirow[t]{2}{*}{ Weight (g) } & \multirow[t]{2}{*}{$\%$} & \multirow[t]{2}{*}{ Weight $(\mathrm{g})$} & \multirow[t]{2}{*}{$\%$} & \multirow[t]{2}{*}{ Weight (g) } & \multirow[t]{2}{*}{$\%$} & \multicolumn{2}{|c|}{ Cloacal } & \multicolumn{2}{|c|}{ Abdominal } & \multicolumn{2}{|c|}{ Total } \\
\hline & & & & & & & & Weight(g) & $\%$ & Weight(g) & $\%$ & Weight(g) & $\%$ \\
\hline \multirow[t]{2}{*}{ Males } & $3,438.6$ & $2,475.4 a^{1}$ & $72.00 \mathrm{a}$ & $743.5 a$ & $30.00 a$ & $718.3 a$ & $29.00 a$ & 49.8 & $2.00 a$ & 24.4 & 1.00 & 74.2 & 3.00 \\
\hline & $3,169.2$ & $2,288.0$ & $72.20 \mathrm{ab}$ & $675.6 \mathrm{a}$ & $29.50 a$ & $668.9 c$ & $29.20 \mathrm{a}$ & 50.3 & $2.20 \mathrm{ab}$ & 25.2 & 1.10 & 75.5 & 3.30 \\
\hline \multirow[t]{2}{*}{ Females } & $2,877.4$ & $2,117.8 b$ & $73.60 \mathrm{~b}$ & $681.2 b$ & $32.20 \mathrm{~b}$ & $582.7 b$ & $27.50 \mathrm{~b}$ & 54.5 & $2.60 a b$ & 27.2 & 1.30 & 81.7 & 3.90 \\
\hline & $2,765.1$ & $2,016.2 d$ & 72.90ab & $619.8 c$ & 30.80ab & $560.0 \mathrm{~b}$ & $27.80 \mathrm{~b}$ & 56.0 & $2.80 \mathrm{~b}$ & 30.5 & 1.50 & 86.5 & 4.30 \\
\hline
\end{tabular}

\begin{tabular}{|c|c|c|c|c|}
\hline \multirow{2}{*}{ Cuts } & \multicolumn{2}{|c|}{ Males } & \multicolumn{2}{|c|}{ Females } \\
\hline & Ross & Avian & Ross & Avian \\
\hline Breast with skin & $1.1 \pm 0.2 \mathbf{a}^{1}$ & $2.1 \pm 0.6 \mathbf{b}$ & $1.1 \pm 0.2 \mathbf{a}$ & $1.8 \pm 0.8 \mathbf{b}$ \\
\hline Leg with skin & $3.8 \pm 0.7$ a & $4.3 \pm 0.5 \mathbf{b}$ & $3.1 \pm 0.5 a$ & $4.1 \pm 0.6 \mathbf{b}$ \\
\hline Skin & $39.6 \pm 4.5 a$ & $36.5 \pm 6.1 \mathbf{b}$ & $50.2 \pm 5.3 \mathbf{b}$ & $54.2 \pm 4.5$ b \\
\hline
\end{tabular}

content was also affected by sex and line. Females generally showed fatter skin, but less cholesterol, than males (Table 6).

Table 7 shows that fatty acid composition of breast and legs according to sex and line. There were no significant statistical differences $(p>0.05)$ among treatments in relation to this parameter, since it is highly dependent of diet. However, differences in the percentage of saturated (SFA), mono (MFA) and polyunsaturated (PFA) fatty acids according to sex and line were detected in fat depots, except for abdominal fat.
Table 6 - Cholesterol content $(\mathrm{mg} / 100 \mathrm{~g})$ in the different cuts of broiler chickens according to sex and line at 50 days of age.

\begin{tabular}{|c|c|c|c|c|}
\hline \multirow[t]{2}{*}{ Cuts } & \multicolumn{2}{|c|}{ Males } & \multicolumn{2}{|c|}{ Females } \\
\hline & Ross & Avian & Ross & Avian \\
\hline Breast with skin & $39 \pm 4 a$ & $45 \pm 5 a b$ & $48 \pm 5$ b & $49 \pm 3 b$ \\
\hline Leg with skin & $85 \pm 1$ a & $77 \pm 11$ a & $81 \pm 8$ a & $84 \pm 9 a$ \\
\hline Skin & $104 \pm 7 a b$ & $112 \pm 5 \mathbf{b}$ & $93 \pm 6 a$ & $98 \pm 4 a$ \\
\hline
\end{tabular}

\section{CONCLUSIONS}

The findings of this experiment showed that birds from Ross line had better performance in the live and carcass parameters; however, if compared only females birds the performance was similar in both lines. On the other hand, the percentage of total fat, saturated, mono and polyunsaturated fatty acids in different tissue were strongly sex and line dependent.

\begin{tabular}{|c|c|c|c|c|}
\hline Cuts & Ross Males & Avian Males & Ross Females & Avian Females \\
\hline \multicolumn{5}{|l|}{ SFA $\%$} \\
\hline Breast w/ skin & $32.3+1.1 a^{1}$ & $29.6+0.8 a$ & $31.7+0.3 c$ & $30.2+1.1 \mathrm{a}$ \\
\hline Leg w/ skin & $30.0+0.9 b$ & $28.0+0.6 b$ & $27.7+0.8 \mathrm{a}$ & $28.6+1.2 a$ \\
\hline Skin & $32.6+1.1 \mathrm{a}$ & $30.3+0.8 \mathrm{cb}$ & $27.9+0.2 b$ & $28.8+1.3 a$ \\
\hline $\begin{array}{l}\text { Abdominal Fat } \\
\text { MFA\% }\end{array}$ & $30.9+1.1 b$ & $29.6+1.1 \mathrm{a}$ & $29.0+0.7 b$ & $28.4+0.9 a$ \\
\hline Breast w/ skin & $33.0+1.1 a$ & $31.6+0.8 a$ & $37.6+0.8 a$ & $34.9+1.2 a$ \\
\hline Leg w/ skin & $33.4+0.9 a$ & $32.5+0.6 a b$ & $36.0+0.5 b$ & $35.8+0.2 a$ \\
\hline Skin & $30.9+1.2 b$ & $32.7+1.0 \mathrm{ab}$ & $35.8+0.6 b$ & $36.4+1.0 \mathrm{a}$ \\
\hline $\begin{array}{l}\text { Abdominal Fat } \\
\text { PFA \% }\end{array}$ & $34.1+1.1 \mathrm{a}$ & $33.6+1.1 b$ & $35.9+0.4 b$ & $36.2+1.2 \mathrm{a}$ \\
\hline Breast w/ skin & $28.5+1.0 \mathrm{ab}$ & $31.8+1.0 \mathrm{a}$ & $27.4+0.6 a$ & $29.4+0.6 a$ \\
\hline Leg w/ skin & $29.7+1.0 \mathrm{a}$ & $31.9+0.9$ a & $26.4+0.7 b$ & $26.4+0.7 b$ \\
\hline Skin & $29.2+1.1 \mathrm{ab}$ & $29.3+1.1 b$ & $28.0+0.2 \mathrm{a}$ & $28.6+0.8 a$ \\
\hline Abdominal Fat & $27.5+0.9 b$ & $29.1+1.1 b$ & $27.5+0.3 a$ & $27.9+0.7 a$ \\
\hline
\end{tabular}

1. For each independent sex, similar letters within a same row are not different statistically $(p>0.05)$. 


\section{REFERENCES}

Ahn DU, Sunwoo HH, Wolfe FH, Sim JS. Effects of dietary alphalinolenic acid and strain of hen on the fatty acid composition, storage stability and flavor characteristics chicken egg. Poultry Science 1995; 74:1540-1547.

Cherian G, Wolfe FW, Sim JS. Dietary oils with added tocopherols: Effects on eggs or tissue tocopherols, fatty acids, and oxidative stability. Poultry Science 1996; 75:423-431.

Folch J, Lees M, Sloane GHS. A simple method for the isolation and purification of total lipids from animal tissues. Journal of Biological Chemistry 1957; 226:497-509.

Garcia PT, Casal J, Parodi J. Effect of breed type on the relationship between intramuscular and total body fat in steers. Meat Science 1999; 17:283-287.

Lázzari GL, Paganini JL. Dimorfismo sexual en el crecimiento muscular y óseo en pollos parrilleros de la línea Cobb 500. Revista de la Faculdad de Agronomia 1999; 19:75-79.

Leeson S. New concepts in feeding broiler chickens. XIV Congreso Latinoamericano de Avicultura. 1995. Chile. p. 30-40.

Mallo G, Melo J, Ciacciariello M, Fernadez P, Miguel G, Villar E. Efectos a la faena de dietas con diferente orígen aminoacídico en dos líneas de pollos parrilleros comerciales. 1997. XV Congreso Latinoamericano de Avicultura. Cancún, Méjico. p. 210-211.

Masato Morita M. Costo beneficio de uso de aceites y grasas en raciones avícolas. Técnicas en Avicultura. 1992. p. 18-22.

Mendes AA, García EA, Inaldo SP. Desempenho e rendimento de carcaças de cinco linhagens comerciais de frango de corte. Boletim Técnico Bigbirds.1988. Ano 1. No I. p. 1-10.

Roschlan P, Bernard E, Gruber W. Enzymatic-colorimetric methods. $9^{\text {th }}$ Congress on Clinical Chemistry. Toronto.1975. Abstract. p. 1.

SAS Institute. Statitical analysis system. User Guide. SAS Institute Inc. Cary, NC. 1998.

Souza PA, Sousa HBA, Campo EF, Brognoni E. Desempeño y características de carcasa de diferentes líneas comerciales de pollos parrilleros. 1995. XIV Congreso Latinoamericano de Avicultura. Chile. p.108-118. 